\title{
Target Zones for Exchange Rates and Policy Changes.
}

\author{
John Driffill ${ }^{a *}$ and Martin Sola ${ }^{a, b}$ \\ ${ }^{a}$ Department of Economics, Birkbeck College, University of London, \\ Malet Street, London WC1E 7HX \\ ${ }^{b}$ Departamento de Economia, Universidad Torcuato Di Tella, \\ Miñnones 2177 (1428) BS. AS., Argentina
}

January 27, 2004

\begin{abstract}
We extend a target zone model to allow for occasional changes in the policy regime which change the stochastic process driving fundamentals. A scenario we have in mind is that macroeconomic policy alternates between relatively tight and loose regimes. A key implication of our analysis is that occurrences which have the appearance of speculative attacks on a currency may be associated with market perceptions of a policy regime switch having taken place. This applies both to a sudden weakening and strengthening of a currency. Our model provides an explanation, based on fundamentals, why large changes in the exchange rate might be associated with no discernible contemporaneous change in the fundamental. Therefore the model provides an explanation for this phenomenon that is an alternative to explanations based on self-fulfilling expectations. Compared with most other models of target zones, other than those relying on intra-marginal intervention, this model is better able to reproduce key features of empirical distributions of exchange rates within the band. The distribution generated by our model has more mass at the centre and less at the edges of the band than is the case for most other models.
\end{abstract}

Keywords: stochastic regime-switching, exchange rates, target zones.

JEL numbers: F31, F41

\footnotetext{
${ }^{*}$ Corresponding author. Acknowledgments: We wish to thank James R. Lothian and an anonymous referee for greatly helping to improve the content and the presentation of the paper. We also want to thank Howard Wall, Ron Smith, Haris Psaradakis, Stephen Hall, and seminar participants at Birkbeck College, Central Bank of Uruguay, Universidad Torcuato Di Tella, Universidad San Andres for comments. The usual disclaimer applies.
} 


\section{Introduction}

A great deal of the literature on exchange rate target zones assumes that the fundamentals driving the exchange rate follow a random walk with drift, and the rate of drift and the variance of innovations in the fundamentals are constant over time (for a survey see e.g. Garber and Svensson (1995)). This of course implies that the characteristics of particular currencies do not change over time: weak currencies (whose fundamentals drift upwards relatively quickly) always tend to be weak (they will tend to drift quickly from bottom to top of the band and be realigned frequently); and strong currencies always tend to be strong. However, there have been fairly frequent and substantial changes in policies, which affect the drift and variability of the stochastic processes driving fundamentals. For example, in the Exchange Rate Mechanism of the European Monetary System there were many realignments between 1979 and 1999. It is unlikely that during this period the governments of ERM participants maintained policies that might be represented by constant drift and variability of fundamentals. The French government in 1982 reversed its expansionary fiscal and monetary stance and adopted tight money aimed at reducing French inflation and establishing a strong Franc. This policy of course culminated in the "Franc fort" policy in 1987, bringing to an end a series of periodic realignments of the Franc against the Deutschemark. The separation of the Bank of Italy from the Treasury in 1981 was a policy change intended, among other things, to strengthen the Lira.

In the context of monetary models, where expectations of future fundamentals affect the current exchange rate, these policy shifts, to the extent that the probability of their occurrence is incorporated into the behavior of agents in the economy, might be expected to have effects on current exchange rates ${ }^{1}$. In particular in a target zone S-curve model, changes in the stochastic processes driving the fundamentals are associated with changes in the steepness of the S-curve, therefore changing the stabilizing or honeymoon effects of the band.

In this paper we treat the fundamentals driving exchange rate movements as a two-state process, allowing the exchange rate to switch randomly from one state (as defined by its rate of drift and innovation variance) to another. This might be thought of as a simple representation of the possibility that policy switches between regimes that are hard and soft from the point of view of fiscal or monetary policy. Regime switches might account for times when exchange rates suddenly come under pressure or appreciate strongly in ways inconsistent with a constant policy regime, and where non-zero probabilities of future policy switches account for an exchange rate being stronger or weaker than would be implied by certain continuation of the current policy. $^{2}$ For example, weak countries might benefit from the stabilizing effect

\footnotetext{
${ }^{1}$ See Sola and Driffill (1994) and Driffill and Sola (1998) for analogous effects on long-term bonds and stock prices respectively.

${ }^{2}$ The analysis here is of potentially recurrent switches of policy regime. In this respect it is to be distinguished from that of, for example, Miller and Sutherland (1991) who explore the implications of a single not-to-be-repeated switch. Our model allows for there having been observed in the data only a single change in regime. It allows for the possibility (but not the necessity) of a greater-than-zero probability of returning to the original regime, that is to say, a peso problem.
} 
of the expectation that their monetary policy would tighten up in the future to meet the needs of the Maastricht convergence criteria or the Stability and Growth Pact.

Our analysis shows that expected changes in the process that drives fundamentals may have dramatic effects on the sustainability of the exchange rate. Depending on the original state of the economy, these changes may result in either a significant gain in the stabilizing effects of the exchange rate band, or an attack-type crisis where the government is forced to conduct a discrete intervention to sustain the band. Our model also provides an explanation, based on fundamentals, for large changes in the exchange rate which are not associated with discernible contemporaneous changes in the fundamentals.

The model analyzed here is general enough to account for changes in regime based on changes in either objective fundamentals or beliefs. The standard models of currency bands are special cases of it. The model also comes closer to replicating the stochastic properties of exchange rate data than most other existing models. It is well known that, even though most models of target zones imply that the exchange rate will remain most of the time at the edges of the band whenever the fundamentals have a drift, in practice exchange rates seem to spend most of their time distributed around the centre of the band. This feature has only been accounted for by models incorporating intra-marginal intervention. However, in those models the role played by the band and the size of the honeymoon effect is smaller. By contrast, our model assigns a large role to the band in stabilizing expectations, and is capable of generating stochastic properties of exchange rates more similar to those found in data. We compare the distributions of exchange rates generated by the model of this paper with distributions produced by a standard one-regime model. While the standard model puts most of the probability distribution near the edges of the band, our model generates an approximately uniform distribution. While this is not entirely consistent with the data, it is much closer to it than are the predictions of the oneregime model. Our model predicts a substantial appreciation of the exchange rate within the band as a result of the reduction of the interest rate differential which took place in the mid eighties; the one-state model predicts only a minor appreciation.

\section{Exchange rates, target zones, and policy changes}

We consider a general model in which there is a devaluation risk that is partly exogenous (the probability of exogenous devaluation follows a random walk with drift following Bertola and Svensson (1993)) and partly a function of the fundamental. As special cases of this there is $(i)$ the possibility that the devaluation risk is purely exogenous, and ( $i i)$ the possibility that the band is fully credible, i.e., the devaluation risk is zero. Each of these may be appropriate according to the circumstances of different countries at different times. ${ }^{3}$ In each of the three scenarios, the monetary

\footnotetext{
${ }^{3}$ Empirical evidence seems to give greatest support to the models with partial credibility of the band (see Svensson, 1992). Campa and Chang (1998) find evidence in favour of models with endogenous realignment probabilities. Garratt, Psaradakis and Sola (2000) also find evidence of non-linearities in models with exogenous realignment probabilities.
} 
policy regime is modelled as switching randomly between two states.

The exchange rate is assumed to be driven by a fundamental $f$, and to satisfy the equilibrium condition

$$
e=f+\alpha E_{t}(d e) / d t
$$

Fundamentals follow a random walk with state dependent drift and variance. We assume that there are two states of the world, 0 and 1, between which it switches from time to time, governed by a Markov process. In state 0 the drift in the fundamental driving process $f$ is $\mu_{0}$ and the variance is $\sigma_{0}^{2}$, while in state 1 the corresponding values are $\mu_{1}$ and $\sigma_{1}^{2}$. The probability of shifting from state 0 to state 1 in an interval of time of length $d t$ is $\theta d t$, and the probability of switching from state 1 to state 0 is $\phi d t .{ }^{4}$ Therefore we may write

$$
\begin{aligned}
d f= & \mu_{0} d t+\sigma_{0} d z \quad \text { in state zero } \\
& \mu_{1} d t+\sigma_{1} d z \quad \text { in state one }
\end{aligned}
$$

The exchange rate $e$ lies within a band with centre $c$ and upper and lower limits $\left(c+\widetilde{x}_{u}, c-\widetilde{x}_{l}\right)$ defended by intervention by the central bank at the margins. The position of the centre of the band may shift from time to time. In each interval of time $d t$, the product of the probability of the occurrence of an exogenous realignment and its size is $g d t$. There is also the possibility of a realignment occurring dependent on the position of the exchange rate within the band: the closer it is to the edge, the more likely is a realignment to occur. This follows Tristani (1994). So the expected movement of the centre of the band is

$$
E(d c)=g d t+\rho(e-c) d t
$$

The exogenous realignment risk $g$ follows a random walk with drift that shifts according to the state of the economy. Thus

$$
\begin{aligned}
d g= & \mu_{g 0} d t+\sigma_{g 0} d z_{g} \quad \text { in state } \\
& \mu_{g 1} d t+\sigma_{g 1} d z_{g} \quad \text { in state one }
\end{aligned}
$$

We assume that, when a devaluation takes place, the actual exchange rate $e$ and the fundamental $f$ shift up by the same amount as does the centre of the band $c$, so that the position of the exchange rate within the band is unchanged - this is an assumption which follows Bertola and Svensson (1993) - and is made largely for convenience. Therefore, expressing movements of the exchange rate around the centre of the band $(e-c)$ we have

$$
\begin{aligned}
e-c & =f-c+\alpha E_{t}(d e) / d t \\
& =f-c+\alpha E_{t}(d(e-c)) / d t+\alpha E_{t}(d c) / d t \\
& =f-c+\alpha E_{t}(d(e-c)) / d t+\alpha(g+\rho(e-c))
\end{aligned}
$$

\footnotetext{
${ }^{4} \mathrm{~A}$ similar idea has been developed in the literature on speculative attacks on fixed exchange rate regimes by Willman (1987) where in the event of a speculative attack the government changes the process governing fundamentals with some probability. For a survey on speculative attacks see Blackburn and Sola (1993). Nevertheless, notice that in the present paper the change in policy is not linked to an actual attack (at which time it is assumed that the band is defended) but may take place at any moment in time.
} 
Defining $\widetilde{x} \equiv e-c$ and $\widetilde{f} \equiv f-c$, the above expression gives

$$
\widetilde{x}=\frac{\widetilde{f}+\alpha g}{1-\alpha \rho}+\frac{\alpha d E_{t}(d \widetilde{x}) / d t}{1-\alpha \rho}
$$

We can define therefore a composite fundamental driving variable $\widetilde{h} \equiv \frac{\widetilde{f}+\alpha g}{1-\alpha \rho}$, and we also define $\widetilde{\alpha}=\alpha /(1-\alpha \rho)$. These allow us to express the equilibrium condition for the foreign exchange market as

$$
\widetilde{x}=\widetilde{h}+\widetilde{\alpha} d E_{t}(d x) / d t
$$

The equation of motion for $\widetilde{h}$ is

$$
\widetilde{d h}=\mu_{h} d t+\sigma_{h} d z
$$

where the drift $\mu_{h}$ and the innovation variance $\sigma_{h}^{2}$ inherit the state-dependency from the parameters of the processes that drive $f$ and $g$. This state-dependence has not been set out explicitly here. We have

$$
\mu_{h}=\left(\mu_{f}+\alpha \mu_{g}\right) /(1-\alpha \rho)
$$

and

$$
\sigma_{h}=\left(\sqrt{\sigma_{f}^{2}+\alpha^{2} \sigma_{g}^{2}+2 \alpha \rho_{f g} \sigma_{f} \sigma_{g}}\right) /(1-\alpha \rho)
$$

where

$$
\rho_{f g}=\operatorname{corr}\left(d z_{f}, d z_{g}\right)
$$

The special cases are obtained by setting the appropriate parameters to zero. When realignments are purely exogenous we have $\rho=0$, when they are purely endogenous $\rho \neq 0$ and $g=0$ with $\mu_{g 0}=\mu_{g 1}=\sigma_{g 0}=\sigma_{g 1}=0$. Perfectly credible target zones are obtained by setting all the above parameters equal to zero.

Although the analysis in this paper is carried out in terms of changes in fundamentals, the model can be used to explain the effects of changes in beliefs about the evolution of realignment risk. One possible situation is that the fundamentals (the money supply, and so on) are the same in both states, and the only difference between states lies in the public's beliefs about realignment risk. In this case a change in beliefs may bring forward the expected date of the next exchange rate "crisis".

\section{Solving for the exchange rate in terms of funda- mentals}

In this section we modify the standard method for solving for the exchange rate in terms of fundamentals, to allow for there being two states of the world. This applies to all three variants of the model presented above. We allow for the fact that whichever state the economy finds itself in today, there is the possibility that it will switch to the 
other state at the end of any small time interval. It is assumed that the public know the current state of the economy and is able to forecast the probability of switching to the other state.

The solution of the asset price equation under state 0 is

$$
e-c=\widetilde{x}_{0}(h)
$$

and under state 1 is

$$
e-c=\widetilde{x}_{1}(h) .
$$

If the economy is known to be in state 0 at time $t$, then at time $t+d t$ the position of the exchange rate within the band is expected to be

$$
E_{t} \widetilde{x}_{t+d t}=E_{t}\left((1-\theta d t) \widetilde{x}_{0}\left(h_{t+d t}\right)+\theta d t \widetilde{x}_{1}\left(h_{t+d t}\right)\right),
$$

and if the economy is known to be in state 1 at time $t$, then at time $t+d t$ the corresponding position will be

$$
E_{t} \widetilde{x}_{t+d t}=E_{t}\left(\phi d t \widetilde{x}_{0}\left(h_{t+d t}\right)+(1-\phi d t) \widetilde{x}_{1}\left(h_{t+d t}\right)\right) .
$$

Then the expected movement of the exchange rate within the band when the economy is in state 0 can be written as

$$
E_{t} d \widetilde{x}_{t+d t}=E_{t}\left(\tilde{x}_{t+d t}-\tilde{x}_{t}\right)=E_{t}\left((1-\theta d t) \widetilde{x}_{0}\left(h_{t+d t}\right)+\theta d t \widetilde{x}_{1}\left(h_{t+d t}\right)-\widetilde{x}_{0}\left(h_{t}\right)\right),
$$

and, analogously, when the economy is in state 1:

$$
E_{t} d \widetilde{x}_{t+d t}=E_{t}\left(\tilde{x}_{t+d t}-\tilde{x}_{t}\right)=E_{t}\left((1-\phi d t) \widetilde{x}_{1}\left(h_{t+d t}\right)+\phi d t \widetilde{x}_{0}\left(h_{t+d t}\right)-\widetilde{x}_{1}\left(h_{t}\right)\right) .
$$

Applying Ito's Lemma we obtain the following equations. Conditional on being in state 0 , the expected change in the exchange rate within the band is

$$
E_{t} d \widetilde{x}_{t+d t}=\left(\mu_{h 0} \widetilde{x}_{0 h}+\frac{\sigma_{h 0}^{2}}{2} \widetilde{x}_{0 h h}+\theta\left(\widetilde{x}_{1}(h)-\widetilde{x}_{0}(h)\right)\right) d t,
$$

while conditional on being state 1 , the expected change in the exchange rate within the band is

$$
E_{t} d \widetilde{x}_{t+d t}=\left(\mu_{h 1} \widetilde{x}_{1 h}+\frac{\sigma_{h 1}^{2}}{2} \widetilde{x}_{1 h h}+\phi\left(\widetilde{x}_{0}(h)-\widetilde{x}_{1}(h)\right)\right) d t .
$$

Then conditional on being in state 0 the differential equation satisfied by the function $\widetilde{x}_{0}(h)$, obtained by substituting from (8) and (9) into (3), is

$$
\widetilde{x}_{0}(h)=h+\alpha\left(\mu_{h 0} \widetilde{x}_{0 h}+\frac{\sigma_{h 0}^{2}}{2} \widetilde{x}_{0 h h}+\theta\left(\widetilde{x}_{1}(h)-\widetilde{x}_{0}(h)\right)\right),
$$

and conditional on being in state 1 the analogous relationship is

$$
\widetilde{x}_{1}(h)=h+\alpha\left(\mu_{h 1} \widetilde{x}_{1 h}+\frac{\sigma_{h 1}^{2}}{2} \widetilde{x}_{1 h h}+\phi\left(\widetilde{x}_{0}(h)-\widetilde{x}_{1}(h)\right)\right) .
$$


The solutions to these equations will lead to a function that expresses the exchange rate in each state as a function of the fundamentals. The deviation of the exchange rate from the central parity $(\widetilde{x})$ lies between lower and upper limits $\underline{x}$ and $\bar{x}$. This requires that the fundamental lies between lower and upper limits in each state: $\underline{h}_{0}$

and $\bar{h}_{0}$ in state 0 and $\underline{h}_{1}$ and $\bar{h}_{1}$ in state 1 . The exchange rate will be an increasing function of the fundamentals between the limits in each state. The fundamentals follow the random walk with drift (specified above) while they remain within the relevant bounds and they are prevented from straying beyond them by means of interventions at the lower and upper limits.

The existence of two states introduces a small complication, in that it is possible for the fundamentals to take values which lie within the range $\left[\underline{h}_{i}, \bar{h}_{i}\right]$ in the current state $i(i=0,1)$, but which lie outside that range for the other state. If there were a change of state, and after this change the fundamentals lie outside the state dependent range, then, the exchange rate would jump to the upper or lower edge of the band as appropriate, and the fundamentals would be brought from their current level to the level of the relevant end-point of the range corresponding to the new state by means of an instantaneous discrete intervention

There are two cases to be distinguished. In the first case the parameters of the model are such that $\underline{h}_{0}<\underline{h}_{1}<\bar{h}_{1}<\bar{h}_{0}$, i.e., state 1 gives rise to a narrow band in the fundamentals and state 0 to a wider band, and the range of fundamentals for state 1 is contained within the range of fundamentals that applies in state 0 . In the second case the parameters are such that the bands overlap. For example, $\underline{h}_{0}<\underline{h}_{1}<\bar{h}_{0}<$ $\bar{h}_{1}$. These two cases cover all the possibilities, and we consider them in turn.

\subsection{Nested Bands}

First we consider a nested bands configuration (see figure 1), in which a wider band brackets a narrower one, with $\underline{h}_{0}<\underline{h}_{1}<\bar{h}_{1}<\bar{h}_{0}$. In this configuration, whenever the fundamentals lie in the range $\left(\bar{h}_{1}, \bar{h}_{0}\right]$ ( which is only possible when the economy is in state 0 ) and a change of state takes place (from state 0 to state 1 ) then, the value of $\widetilde{x}$ increases from its position in state $0\left(\widetilde{x}_{0}(h)\right)$ to the upper edge of the band $(\bar{x})$. This requires an immediate discrete intervention to reduce the value of the fundamentals to the level corresponding to the upper limit in state $1\left(\bar{h}_{1}\right)$.

This has the following consequences for the solutions to the equations (10) and (11) above. For values of $h$ within the range $\left[\underline{h}_{1}, \bar{h}_{1}\right]$, the solutions to $(10)$ and (11) above define functions $F($.$) and G($.$) such that in state 0 \widetilde{x}_{0}(h)=F(h)$ and $\widetilde{x}_{1}(h)=G(h)$. For values of $h$ in the range $\left(\bar{h}_{1}, \bar{h}_{0}\right]$, equation (10) above, with $\widetilde{x}_{1}(h)=\bar{x}$, defines a function $D^{U}($.$) such that \widetilde{x}_{0}(h)=D^{U}(h)$. Analogously, for values of $h$ within the range $\left[\underline{h}_{0}, \underline{h}_{1}\right)$, equation $(10)$ with $\widetilde{x}_{1}(h)=\underline{x}$ defines a function $D^{L}($.$) such that \widetilde{x}_{0}(h)=D^{L}(h)$.

In summary, the exchange rate in each state is expressed as follows:

$$
\widetilde{x}_{0}(h)=\left\{\begin{array}{l}
D^{L}(h), \underline{h}_{0} \leq h<\underline{h}_{1} \\
F(h), \underline{h}_{1} \leq h \leq \bar{h}_{1} \\
D^{U}(h), \bar{h}_{1}<h \leq \bar{h}_{0}
\end{array}\right\}
$$




$$
\widetilde{x}_{1}(h)=G(h), \underline{h}_{1} \leq h \leq \bar{h}_{1} .
$$

Over the range $\left[\underline{h}_{1}, \bar{h}_{1}\right]$, the solution for the exchange rate is

$$
\begin{aligned}
& F(h)=\widetilde{x}_{0}(h)=\Sigma_{i=1}^{4} A_{i} e^{\lambda_{i} h}+h+\frac{\alpha\left[\mu_{h 0}(1+\alpha \phi)+\mu_{h 1} \alpha \theta\right]}{1+\alpha \phi+\alpha \theta}, \\
& G(h)=\widetilde{x}_{1}(h)=\Sigma_{i=1}^{4} B_{i} e^{\lambda_{i} h}+h+\frac{\alpha\left[\mu_{h 1}(1+\alpha \theta)+\mu_{h 0} \alpha \phi\right]}{1+\alpha \phi+\alpha \theta},
\end{aligned}
$$

where the $\lambda^{\prime} s$ are the roots which solve the homogeneous part of the system of differential equations (10) and (11), which have trial solutions $\widetilde{x}_{0}(h)=A e^{\lambda h}$ and $\widetilde{x}_{1}(h)=$ $B e^{\lambda h}$ with $A_{i}$ and $B_{i}$ determined by the terminal conditions. (A detailed explanation of the solution procedure and the terminal conditions is presented in the Appendix.)

Over the range $\left[\underline{h}_{0}, \underline{h}_{1}\right)$, the solution for the exchange rate is

$$
D^{L}(h)=\frac{\alpha \theta \underline{x}+\alpha \mu_{0 h}(1+\alpha \theta)^{-1}}{(1+\alpha \theta)}+\frac{1}{(1+\alpha \theta)} h+D_{1}^{L} e^{\delta_{1}^{L} h}+D_{2}^{L} e^{\delta_{2}^{L} h},
$$

where the $\delta^{\prime} s$ are the roots which solve the homogenous part of the differential equation defined by equation (10) replacing $\widetilde{x}_{1}(h)$ by $\underline{x}$. This equation has trial solutions $\widetilde{x}_{0}(h)=D^{L}(h)$ with the constants of integration $D_{1}^{L}$ and $D_{2}^{L}$ chosen so as to satisfy the boundary conditions.

Over the range $\left(\bar{h}_{1}, \bar{h}_{0}\right]$ the solution for the exchange rate is

$$
D^{U}(h)=\frac{\alpha \theta \bar{x}+\alpha \mu_{0 h}(1+\alpha \theta)^{-1}}{(1+\alpha \theta)}+\frac{1}{(1+\alpha \theta)} h+D_{1}^{U} e^{\delta_{1}^{U} h}+D_{2}^{U} e^{\delta_{2}^{U} h},
$$

with $\delta_{1}^{U}=\delta_{1}^{L}$, and $\delta_{2}^{U}=\delta_{2}^{L}$.

These functions have to satisfy appropriate smooth-pasting and value-matching conditions. At the upper and lower points of the range for fundamentals in state 1 (the narrower range), it must be true that $G\left(\underline{h}_{1}\right)=\underline{x}$, and $G\left(\bar{h}_{1}\right)=\bar{x}$, and that $G^{\prime}\left(\underline{h}_{1}\right)=0$, and $G^{\prime}\left(\bar{h}_{1}\right)=0$. For state 0 (the wider range), it must be true at the end points (in terms of both the fundamentals and the exchange rate) that $D^{L}\left(\underline{h}_{0}\right)=\underline{x}$ and $D^{H}\left(\bar{h}_{0}\right)=\bar{x}$, and that $D^{L \prime}\left(\underline{h}_{0}\right)=0$ and $D^{H^{\prime}}\left(\bar{h}_{0}\right)=0$. Within state 0 , it must additionally be true that, at the end-points of the range of fundamentals in state $1, \underline{h}_{1}, \bar{h}_{1}$, the function $F($.$) has to have the same level (value matching) and slope$ (smooth pasting) as the functions $D^{L}($.$) and D^{U}($.$) , that is to say F\left(\underline{h}_{1}\right)=D^{L}\left(\underline{h}_{1}\right)$ and $F^{\prime}\left(\underline{h}_{1}\right)=D^{L^{\prime}}\left(\underline{h}_{1}\right)$, and $F\left(\bar{h}_{1}\right)=D^{U}\left(\bar{h}_{1}\right)$ and $F^{\prime}\left(\bar{h}_{1}\right)=D^{U^{\prime}}\left(\bar{h}_{1}\right)$.

\subsection{Overlapping Bands}

It is also possible that the range of fundamentals covered by the two states are overlapping (see figure 2), i.e., $\underline{h}_{0}<\underline{h}_{1}<\bar{h}_{0}<\bar{h}_{1}$. Under this configuration whenever the fundamentals lies in the range $\left[\underline{h}_{0}, \underline{h}_{1}\right.$ ) (which can only take place if the economy is in state 0 ), and that the economy switches from state 0 to state 1 , then, the exchange 
rate $\widetilde{x}_{0}(h)$ jumps to the lower edge of the band $\underline{x}$, and the fundamentals have to be increased to $\underline{h}_{1}$ by means of a discrete intervention. Alternatively, suppose that the economy is in state 1 , with the fundamentals $h$ in the range $\left(\bar{h}_{0}, \bar{h}_{1}\right]$, and suppose that there is a switch of state from state 1 to state 0 . Then, the exchange rate $\widetilde{x}_{1}(h)$ jumps to the top of its band, $\bar{x}$, and the fundamentals have to be decreased to $\bar{h}_{0}$ by means of a discrete intervention. If the fundamentals lie in the range of values spanned in both states, that is $\left[\underline{h}_{1}, \bar{h}_{0}\right]$ then the exchange rate will jump from the solution in one state to the solution in the other, that is, between $\widetilde{x}_{0}(h)$ and $\widetilde{x}_{1}(h)$, with no interventions.

The consequences for the solutions to the equations (10) and (11) are that for $h$ in the range $\left[\underline{h}_{1}, \bar{h}_{0}\right]$, the solutions define functions $F(h)$ and $G(h)$ with $\widetilde{x}_{0}(h)=F(h)$ and $\widetilde{x}_{1}(h)=G(h)$. For $h$ in the range $\left[\underline{h}_{0}, \underline{h}_{1}\right)$, we solve the equation that results from replacing $\widetilde{x}_{1}(h)$ by $\underline{x}$ in equation $(10)$, and this solution defines a function $\widetilde{x}_{0}(h)=$ $D^{L}(h)$. For $h$ in the range $\left(\bar{h}_{0}, \bar{h}_{1}\right]$, we obtain the solution to (11) once we replaced $\widetilde{x}_{0}(h)$ by $\bar{x}$, and this solution defines a function $\widetilde{x}_{1}(h)=D^{U}(h)$.

Thus the exchange rate in each state is

$$
\begin{aligned}
& \widetilde{x}_{0}(h)=\left\{\begin{array}{c}
D^{L}(h), \underline{h}_{0} \leq h \leq \underline{h}_{1} \\
F(h), \underline{h}_{1} \leq h \leq \bar{h}_{0}
\end{array}\right\} \\
& \widetilde{x}_{1}(h)=\left\{\begin{array}{c}
G(h), \underline{h}_{1} \leq h \leq \bar{h}_{0} \\
D^{U}(h), \bar{h}_{0} \leq h \leq \bar{h}_{1}
\end{array}\right\}
\end{aligned}
$$

Notice that the functions $F(h)$ and $G(h)$ are defined over the same range as in the nested configuration. Therefore they should be solved in exactly the same way. Their solutions are given by equations (12) and (13).

The solution for $D^{L}(h)$ is obtained by solving the equation which arises from replacing $\widetilde{x}_{1}(h)$ by $\underline{x}$ in equation (10), and the solution is

$$
D^{L}(h)=d_{a}^{L}+d_{b}^{L} h+D_{1}^{L} e^{\delta_{1}^{L} h}+D_{2}^{L} e^{\delta_{2}^{L} h}
$$

in which $d_{a}^{L}, d_{b}^{L}, \delta_{1}^{L}$ and $\delta_{2}^{L}$ are defined as in the nested case and $D_{1}^{L}$ and $D_{2}^{L}$ are constants of integration to be determined by boundary conditions.

The solution for $D^{U}(h)$ is obtained by solving equation $(11)$ with $\widetilde{x}_{0}(h)$ replaced by $\bar{x} .^{5}$

Following the procedure outlined in the Appendix, it is easy to show that the general solution for $D^{U}(h)$ is

$$
D^{U}(h)=d_{a}^{U}+d_{b}^{U} h+D_{1}^{U} e^{\delta_{1}^{U} h}+D_{2}^{U} e^{\delta_{2}^{U} h}
$$

where $d_{a}^{U}=\alpha \frac{\phi \bar{x}+\mu_{0 h}(1+\alpha \phi)^{-1}}{(1+\alpha \phi)}, d_{b}^{U}=\frac{1}{(1+\alpha \phi)}, \delta_{1}^{U} h$ and $\delta_{2}^{U} h$ are the roots of the solution of the homogeneous part of equation (11) with $\widetilde{x}_{0}(h)=\bar{x}$, and $D_{1}^{U}$ and $D_{2}^{U}$ are the constants of integration, chosen so as to satisfy the boundary conditions.

\footnotetext{
${ }^{5}$ This yields the following differential equation $[1+\alpha \phi] \widetilde{x}_{1}(h)=h+\alpha\left(\mu_{h 1} \widetilde{x}_{1 h}+\frac{\sigma_{h 1}^{2}}{2} \widetilde{x}_{1 h h}+\phi \bar{x}\right)$.
} 
As in the case of nested bands, these functions have to satisfy the appropriate smooth pasting and value matching conditions. At the upper and lower values of the ranges of fundamentals in each state, the exchange rate has to take values equal to those at the edges of the band, and the slope of the functions has to equal zero to satisfy smooth pasting. Therefore $D^{L}\left(\underline{h}_{0}\right)=\underline{x}$ and $D^{L \prime}\left(\underline{h}_{0}\right)=0$. $D^{U}\left(\bar{h}_{1}\right)=\bar{x}$ and $D^{U^{\prime}}\left(\bar{h}_{1}\right)=0$. At the point $\underline{h}_{1}$ where the functions $D^{L}(h)$ and $F(h)$ join, they satisfy value matching and smooth pasting conditions: $D^{L}\left(\underline{h}_{1}\right)=F\left(\underline{h}_{1}\right)$ and $D^{L^{\prime}}\left(\underline{h}_{1}\right)=F^{\prime}\left(\underline{h}_{1}\right)$. Similarly for $G(h)$ and $D^{U}(h)$, we have: $G\left(\bar{h}_{0}\right)=D^{U}\left(\bar{h}_{0}\right)$ and $G^{\prime}\left(\bar{h}_{0}\right)=D^{U^{\prime}}\left(\bar{h}_{0}\right)$.

\section{Calibration and Evaluation of the Model}

To calibrate the model we use data for France/Germany 1979-1992. We $i$ ) use daily data and obtain the implied fundamentals using the drift adjustment method, which gives rise to the nested bands configuration and $i i$ ) use quarterly data and derive the fundamentals using a monetary model of exchange rate determination, which gives rise to the overlapping bands configuration ${ }^{6}$. Note that from a theoretical point of view there are no a priori reasons to chose one modelling strategy rather than the other. Interestingly, the use of these two alternative definitions of fundamentals gives rise to the two configurations analyzed in the paper.

\subsection{Nested Bands}

The nested configuration is likely to occur when the two regimes have similar rates of drift and very different variances, since the option value implicit in the band and therefore the strength of the honeymoon effect, are positively correlated with the variance of the innovations in the fundamentals. In this section we explore a numerical example with these properties.

We calibrate the model and calculate the relationships between the exchange rate and fundamentals that it implies. We take the following values: $\alpha=1.471, \mu_{h 0}=$ $0.4869, \mu_{h 1}=0.081, \sigma_{h 0}=19.944, \sigma_{h 1}=4.306, \phi=0.034, \theta=0.188 .{ }^{7}$ The roots $\left(\lambda_{1}, ., \lambda_{4}\right)$ associated with the functions $F($.$) and G($.$) are -0.282,-0.066,+0.064,+0.273$, while

\footnotetext{
${ }^{6}$ We use the standard drift-adjustment approach of Bertola and Svensson (1993) to obtain "drift adjusted" fundamentals. We followed Flood et al. (1991) in constructing a measure for fundamentals as $h_{t}=e_{t}-\alpha \delta_{t}$, where $\delta_{t}$ is a suitable (discretely sampled) interest-rate differential. Our estimate of $\alpha$ used in the calculations was 1.471. A measure of $h_{t}^{*}$ was then obtained as $h_{t}^{*}=h_{t}-c_{t}+\alpha g_{t}$, with the expected rate of devaluation $g_{t}$ being calculated as the deviation of the expected within-band depreciation - estimated as in Rose and Svensson (1995) - from the interest rate differential.

${ }^{7}$ For these values we draw on Driffill and Sola (1999) in which models for the French Franc/ Deutschemark exchange rate are estimated using the drift-adjustment procedure and data sampled every two days for the period March 1979 to September 1992. In many papers in this area, values of $\alpha$ lying between 0.1 and 1.0 have been used, with the majority of examples in the range 0.5 to1. We have experimented with these lower values of $\alpha$ without our results being materially affected. Different values for $\mu_{h 0}, \mu_{h 1}, \sigma_{h 0}, \sigma_{h 1}$ will also leave the qualitative implications of the analysis unchanged as shown in section 5 .
} 
the roots $\left(\delta_{1}, \delta_{2}\right)$ associated with the functions $D^{L}($.$) and D^{U}($.$) are -0.0672$ and 0.0648 .

We computed the relationships between the fundamentals and the exchange rate in each state and found that the thresholds for the fundamentals which keep the exchange rate within the bands are $\left(\underline{h}_{0}=-2.2327, \bar{h}_{0}=2.2245\right)$ in state 0 and $\left(\underline{h}_{1}=-0.803, \bar{h}_{1}=0.8068\right)$ in state 1 . These relationships are shown in figure $1^{8}$.

\subsubsection{Changes in regime and interventions by the Central Bank}

The intervention of the Central Bank can be marginal or discrete. Discrete interventions are associated with changes in the state of the economy that force the central bank to intervene to maintain the exchange rate within the bands while marginal interventions take place at the relevant state dependent thresholds.

For example if the economy is in state 0 and the fundamentals decrease to $\underline{h}_{0}=$ -2.2327 , then the Central Bank has to (marginally) intervene to keep the exchange rate in the band. Marginal intervention also takes place at the edges of the band when the economy is in state 1 and the fundamentals lie in the range $\left(\underline{h}_{1}=-0.803, \bar{h}_{1}=\right.$ $0.8068)$ or if the economy is in state 0 and fundamentals lie in the range $\left(\bar{h}_{1}=\right.$ $\left.0.8068, \bar{h}_{0}=2.2245\right)$.

Changes in the policy regime may have very different immediate effects on fundamentals and on the exchange rate. There are two cases which deserve special attention.

i) Changes in Regime that trigger a discrete intervention by the Central Bank

Under certain circumstances a change in regime forces the exchange rate all the way to the edge of the band and may also require intervention to hold it there, which requires a change in fundamentals. In figure 1 we show that, a change from state 0 to state 1 when the fundamentals are at $h^{(i i i)}$, will require a change in fundamentals to $h^{(i i)}$ to keep the exchange rate at the edge of the band.

This case is interesting because the regime switches mimic speculative attacks which force discrete intervention by the Central Bank as in Flood and Garber (1991). ${ }^{9}$

\footnotetext{
${ }^{8}$ Notice that given that the volatility is greater in state 0 than in state 1 , the corresponding thresholds for the fundamentals are much wider in state 0 than in state 1 . Notice as well that even though the range is wider in state 0 than in state 1 , this does not imply that the fundamentals will hit the threshold later in state 0 than in state 1 , because the drift is greater in state 0 . We calculated the expected time taken for the exchange rate to hit the upper edge of the band (the expected time taken to reach the upper edge of the band starting from the mid-point is $\bar{h}_{i} / \mu_{i}$ (Cox and Miller, 1965, page 220.)) and found that $\bar{h}_{0} / \mu_{0}=2.22 / 0.48<\bar{h}_{1} / \mu_{1}=0.80 / 0.081$. This shows (conditional on the economy remaining in the same state) that the exchange rate will hit the band sooner in state 1.

${ }^{9}$ Note that the discrete jump in the fundamentals generated by this model differs from an apparently similar phenomenon in Flood and Garber (1991) in that here the jump is unpredictable and may occur at any value of the fundamentals whereas in Flood and Garber's analysis it happens predictably when the fundamentals reach their limits. Here the discrete interventions are such that the expected devaluation associated with them are zero. As Flood and Garber (1991) remark in their paper, the discrete intervention, which takes the fundamentals from a point between $\bar{h}_{0}$ and $\bar{h}_{1}$ to $\bar{h}_{1}$ itself, in Figure 1, may be interpreted as being a response to a speculative attack.
} 
Notice as well that such intervention might be triggered by an attempt by the Central Bank to shift its monetary policy towards one which involves smaller variability of the fundamentals and possibly also a lower rate of drift (that is a change from state 0 to state 1). This may induce a depreciation of the currency and indeed discrete intervention may be needed to keep the exchange rate in the band. This will result in large interventions by Central Banks without large changes in the exchange rate. ${ }^{10}$

ii) Changes in regime that stabilize the exchange rate and leave fundamentals unchanged

Here we analyze the situation under which a change in regime causes the exchange rate to jump, without there being a change in fundamentals. Using figure 1 we can see that this case takes place when fundamentals are for example at $h^{(i)}$ and a switch of state takes place. This change in the state of the economy causes, depending on the initial state, either an appreciation or a depreciation of the exchange rate leaving the fundamentals unchanged. ${ }^{11}$

Notice that this provides an explanation of large movements in the exchange rate with no immediate associated changes in fundamentals that is an alternative to explanations based on self-fulfilling expectations (e.g., Obstfeld 1986, 1996). ${ }^{12}$

In terms of our model there are ranges where regime-switches or policy changes do not involve a change in the level of the fundamentals (for example, the money supply) and jumps in the exchange rate are likely to be associated with no discernible contemporaneous change in the fundamental. In particular, if the economy is in state 1 , with fundamentals therefore in the range $\left(\underline{h}_{1}=-0.803, \bar{h}_{1}=0.8068\right)$, and switches to state 0 , the exchange rate appreciates for relatively high fundamentals (i.e., when the exchange rate is relatively weak) and depreciates for relatively low values (i.e., when the exchange rate is relatively strong). Switching in this direction moves the exchange rate towards the middle of the band and thus helps to maintain the target zone. By contrast, if the economy is in state 0 and the fundamentals are in the range $\left(\underline{h}_{1}=-0.803, \bar{h}_{1}=0.8068\right)$, and the state switches to state 1 , the exchange rate will move towards the edge of the band. Thus such regime switches will lead to what appears to be an exchange rate crisis or a speculative attack on the currency ${ }^{13}$.

\footnotetext{
${ }^{10}$ This has the empirical implication that attempts to explain jumps in the exchange rate by current and past fundamentals may therefore fail or produce misleading results.

${ }^{11}$ The same kind of event occurs in Bertola and Svensson (1993) when there is a discrete jump in the expected rate of devaluation, which they model as a Poisson process, and which is not explicitly connected with any other events. They explore the consequences of fluctuating devaluation expectations but provide no explanation for them. Here, by contrast, discrete jumps in the exchange rate are associated with changes in policy regime.

${ }^{12}$ Self-fulfilling expectations may occur when the attack triggers a change of policy, such as a switch from a constant to a growing money supply, which validates the attack. Empirically, these alternatives are difficult to distinguish, since in both cases the current value of the fundamentals does not change materially at the time of the jump in the exchange rate.

${ }^{13}$ The interpretation of the jump in the exchange rate associated with a regime switch is slightly different depending on the original model, i.e., the fully credible target zones, the exogenous realignment probabilities or the endogenous realignment probabilities model. In the endogenous realigment model, the effect of the change in regime, in shifting the exchange closer to or further away from the edge of the band, is to change the realignment risk discretely. When the exchange rate is shifted towards the upper edge of the band (in terms of $\widetilde{x}$ ) then the realignment risk is increased.
} 
Sensitivity Analysis In this section we assess the sensitivity of the upper and lower values of the fundamentals (at which the exchange rate reaches the upper and lower edges of the band) to changes in key parameters. The results are presented in table 1 and show that as $\sigma_{h 0}$ increases (the variability of the fundamentals in state 0 ), the fundamentals bandwidth also increases in state 0 and in state 1 . As a result, the "s-shaped" curve becomes flatter and therefore the honeymoon effect becomes stronger. When $\sigma_{h 1}$ is increased (the low-variance state) the fundamentals bandwidth also increases in both states.

The effects of an increase in, $\mu_{h 0}$ is to weaken the currency in both states since there is a decrease of the range of fundamentals consistent with the band in both states. The effect on the currency in state 0 is a direct effect while the effect on the currency in state 1 is indirect (and of much smaller magnitude) and it arises because, for any given value of the fundamentals, a shift of regime to state 0 would be associated with a larger depreciation of the currency. Similar results obtain for changes in $\mu_{h 1}$.

The persistence of the regimes (and therefore the probability of changing regimes) measured by the transition probabilities might be a reflection of changes in governments, or un-modelled policy changes, such as changes in the government's fiscal policies which in turn will affect future monetary growth. We find that when the probability of remaining in state $0\left(q=\frac{1}{1+\theta}\right)$ is increased, the fundamentals bandwidth for both state 0 and state 1 widens, since state 0 has the highest volatility. Given that state 1 has the smallest volatility, it is not surprising that an increase in the probability of staying in state $1\left(p=\frac{1}{1+\phi}\right)$ has the effect of narrowing the width of the band in terms of fundamentals in both states.

\subsection{Overlapping Bands}

Overlapping bands are likely to be found when the two states are characterized by substantially different drifts and similar variances. We use quarterly data for France and Germany for the period 1979q1 to $1992 \mathrm{q} 3$ to estimate the relevant parameters. In this section we assume that the evolution of the fundamentals is based on the monetary model of exchange rate determination (on their relative money supplies and real GDPs). The fundamentals are defined as $f_{t} \equiv m_{t}-m_{t}^{*}-\left(y_{t}-y_{t}^{*}\right)$ where $m_{t}$ is the log of the German money supply, $m_{t}^{*}$ the log of the French money supply, and so on ${ }^{14}$. It is assumed that the first difference of the fundamentals $\Delta f_{t}$ is distributed $N\left(\alpha_{0}, w_{0}^{2}\right)$ when $s_{t}=0$, and $N\left(\alpha_{1}, w_{1}^{2}\right)$ when $s_{t}=1$. The evolution of the fundamentals is modelled as a two-state random walk with drift

$$
f_{t+1}=f_{t}+\alpha_{0}\left(1-s_{t+1}\right)+\alpha_{1} s_{t+1}+\left(w_{0}\left(1-s_{t+1}\right)+w_{1} s_{t+1}\right) \varepsilon_{t+1}
$$

with $\operatorname{var}\left(\varepsilon_{t}\right)=1$. The states are assumed to evolve as a first-order homogeneous Markov process with $\operatorname{prob}\left(s_{t}=1 \mid s_{t-1}=1\right)=p$, and $\operatorname{prob}\left(s_{t}=0 \mid s_{t-1}=0\right)=q$. The estimates of the parameters are presented in Table 2. We find that the estimated variances are roughly the same size and that the drift is positive in state 0 while it

\footnotetext{
${ }^{14}$ We have experimented with alternative values for the income elasticity of the demand for money in the range $[1,2]$ and the results were not materially altered.
} 
is negative in state 1 . The estimated transition probabilities are approximately 98 percent which shows that both states are very persistent. The first difference in the value of the fundamentals is plotted in Figure 3, along with the filtered probabilities of being in state 1 and it shows that the economy was in state 0 until 1987 and thereafter in state 1. The clear decline in the expansion of the fundamentals which takes place around 1987 might be associated with the introduction of the 1987's 'Franc fort' policy.

We convert the estimated parameters, which are based on a discrete-time model with quarterly observations, to values appropriate to the corresponding continuoustime model (where the time unit is on an annual basis), as follows. $\mu_{0}=400\left(\alpha_{0}+\right.$ $\left.w_{0}^{2} / 2\right)=3.28, \mu_{1}=400\left(\alpha_{1}+w_{1}^{2} / 2\right)=-7.6534 .1, \sigma_{0}^{2}=\left(400 w_{0}\right)^{2}=37.3, \sigma_{1}^{2}=$ $\left(400 w_{1}\right)^{2}=34.1, \phi=\left(1-p^{4}\right) / p^{4}=0.021, \theta=\left(1-q^{4}\right) / q^{4}=0.019 .^{15}$

Using the above parameters and solving for the relations between the fundamentals and the exchange rate in each of the two states we find that this case corresponds to the case set out above for the overlapping bands. ${ }^{16}$ The roots in the solutions for the functions $F($.$) and G($.$) are 0.527,-0.295,-0.0781$, and 0.124 . The roots in the solution for the function $D^{L}($.$) are 0.124$ and-0.295, and those for $D^{U}($.$) are 0.527$ and -0.0781. The limits of the values of the fundamentals in each state do overlap and are found to be are, for state 0 [-1.083, 0.965], and for state $1[-0.847,1.131]$. The relation between the exchange rates and the fundamentals are graphed in Figure 2.

Sensitivity analysis The results reported in table 3 are qualitatively similar to those reported in table 2 . The main difference arises when we analyze the effects of increasing the transition probabilities. Whereas in the nested case they either enlarged or narrowed the bands (because the states differed mainly in their volatility) here the effect is to shift the whole band (for the fundamentals) either to the right or left (because the volatilities are roughly the same and the main difference between the states comes from the drift parameter). If, for example, the probability of staying in state 1 is increased, then both ranges shift to the right strengthening the exchange rate (the drift in state 1 is negative, see figure 2 ).

\section{$5 \quad$ Empirical properties of exchange rates predicted by the model}

\subsection{Exchange rate distribution}

In this section we assess to what extent the model is capable of reproducing the empirical properties of the exchange rate within the band. It is well known that

\footnotetext{
${ }^{15}$ We have also estimated a single-regime model for the fundamental for comparison purposes and found that: $\mu=-1.63, \sigma^{2}=66.6$. Notice that in the one-state model the drift is slightly negative and the variance is larger than the variance for either of the two regimes in the two-state case. We have found that the range of the fundamentals consistent with the band is $[1.25,-1.21]$.

${ }^{16}$ This is to be expected since the two states differ in their drifts and hardly at all in the variances, suggesting that the s-shaped curve for one state would effectively be a horizontally transposed version of that for the other state.
} 
(whenever the fundamentals have a drift different from zero) the one state model fails to replicate the stochastic properties of the data because the simulated exchange rate would spend most of the time on the upper or lower edge of the band (depending on the sign of the drift). Here we evaluate the stochastic properties of the data generated by the two states model under the two possible configurations and calibrations. These configurations have very different properties.

For the nested configuration (and for our calibrated parameters) both drifts have the same sign, and therefore changes in regime will only change the speed at which the exchange rate converges to the edge of the band. On the other hand, for the overlapping configuration the calibrated means have opposite signs. Then a change in the state changes the direction of expected movement of the exchange rate from appreciation to depreciation (and vice versa). This tends to keep the exchange rate in the middle of the band. We discuss both cases below.

For the nested configuration we carried out a simulation (not reported) of the two-state model which yields modest improvements over the one-state model. This is a consequence of the drift of fundamentals being positive in both states. For this case most of the mass of probability of the exchange rate distribution lies at one edge of the band.

When we simulate the movements of the exchange rate within the band for the overlapping configuration we obtain results that are more consistent with the empirical regularities of the exchange rates. To generate the empirical distributions using the model we adjusted the parameters to correspond to daily observations. Each simulation has 3510 daily observations, corresponding to the sample period, and we take 1000 replications. ${ }^{17}$

We present in figure 4 histograms of the actual data, the generated data using the one-state model and the two-state model under the overlapping configuration. We also inquire how the histograms of the data generated by the two-state model are affected by changes in the relevant parameters. Therefore we also present histograms of data generated by the model under different scenarios (higher mean or variance in each state). The main finding is that for our calibration the one-state model is unable to replicate the properties of the data and has most of the probability mass close to the edge of the band. On the other hand the two-state model has more of the mass of probability near the center of the band, even though it has higher mass at the edges than does the empirical data. When we compare the empirical distributions of the generated data modifying the key parameters of the model (we doubled each parameter in turn) we find that the modification of the shape of the distribution is minor. ${ }^{18}$

\footnotetext{
${ }^{17}$ To carry out the empirical simulations we reflect the fundamentals by .3 (regime dependent) standard deviations. For values in the range of $\{0.1,0.5\}$ standard deviations, the results remain unaffected yielding empirical distributions with similar (uniform) shape. For higher values of the reflecting parameters the distribution looks more bell shaped, and for smaller values it accumulates a higher mass of probabilities at both edges

${ }^{18} \mathrm{An}$ important point to have in mind is that the one state model can only replicate the empirical properties of the exchange rate if the drift is zero, while there are many possible configurations for both the embedded and overlapping cases that can replicate the properties of the data. Even
} 


\subsection{Interest rates differential and the evolution of the ex- change rate within the band.}

In this section we use quarterly data for France and Germany for the period 1979q1 to $1992 \mathrm{q} 3$ to asses the relative strength of the exchange rate within the band. We show in the right-hand panel of figure 5 the evolution of the interest rate differential and exchange rate predicted by the one-state model and the two-state model under the nested configuration. The plots show that the exchange rate within the band strengthened considerably after the substantial reduction in interest rates of the mideighties, which was a result of the "Franc fort" policy. Notably the two-state model predicts a dramatic strengthening of the exchange rate as a result of the economy moving to a new state (economic environment) while the one-state model predicts only a modest appreciation. In the left-hand panel of the figure we plot the parity exchange rate and the actual exchange rate over the same period. After the shift of regime to state 1 in 1986, there were no further realignments in of the exchange rate band. This shows the importance of allowing for changes in the processes that drive fundamentals in models of exchange rate bands. ${ }^{19}$

\section{Conclusions}

In this paper we have extended a target zone model to allow for occasional changes in the policy regime which change the stochastic process driving fundamentals. These changes of policy are modeled as non-zero-probability events. A scenario we have in mind is that macroeconomic policy alternates between relatively tight and loose regimes. We analyze the model both in terms of its theoretical implications and in terms of its ability to reproduce the empirical properties of the exchange rates.

Our analysis shows that movements in the exchange rate which have the appearance of speculative attacks on a currency may be associated with market perceptions of a policy-regime switch having taken place. This applies both to a sudden weakening and strengthening of a currency. The exchange rate will weaken and may require discrete intervention to sustain the band whenever the exchange rate is relatively weak and there is a shift from the regime characterized by a wide band in terms of fundamentals to the regime characterized by a narrow band in terms of fundamentals. On the other hand, if the exchange rate were relatively strong, the same regime shift would cause the currency to strengthen and it might have to be held within the band by intervention, in this case involving large sales rather than purchases.

though in this paper we do not attempt to answer the question of which parameter values can better mimic the empirical exchange rate distribution (and prefer to use calibrations which are consistent with the data), this exercise can be successfully carried out for a range of parameter configurations.

${ }^{19}$ Although the exchange rate became stable for six years after the last realignment in 1986 (as a result of the change in fundamentals), the level was in fact above the central parity for most of the period. Our model, in common with all others, assumes that at the time of realignment the change in the central parity is such that the exchange rate does not move within the band. However, in practice the actual change in parity may not have fulfilled this assumption on this occasion. The crucial thing is that the exchange rate stabilized after the change in fundamentals. 
Our model can also account for large changes in exchange rates with no immediate change in the level of fundamentals, and it provides an alternative theoretical explanation for this phenomenon to that based on self-fulfilling expectations.

The model is also capable of accounting for empirical properties of the exchange rate that simple models with one state and without intra-marginal intervention cannot. We find that the empirical distributions are closer to the distribution of actual data and that the exchange rate within the band co-moves with interest rate expectations.

Notice that these results show that the one-state and the two-state models have very different implications for the usefulness of the target zone as a stabilizing device. When there are intra-marginal interventions the exchange rate tends to stay away from the edges of the band and the honeymoon effect is diminished. Our model assigns a major role to the bands in stabilizing the exchange rate.

\section{References}

[1] Bertola, G., and L. E. O. Svensson, 1993, "Stochastic Devaluation Risk and the Empirical Fit of Stochastic Target Zone Models", Review of Economic Studies, $60,689-712$

[2] Blackburn, Keith, and Martin Sola, 1993, "Speculative Currency Attacks and Balance of Payments Crises," Journal of Economic Surveys, 7, 119-144.

[3] Campa, J. M., and P. H. K. Chang, 1998, "ERM Realignment Risk and its Economic Determinants as reflected in Cross-Rate Options," Economic Journal, 108, 1046-1066.

[4] Cox, D. R., and H. D. Miller, 1965, The Theory of Stochastic Processes, Chapman and Hall.

[5] Driffill, J., and Martin Sola, 1998, "Intrinsic Bubbles and Regime Switching," Journal of Monetary Economics, 42, 257-373.

[6] Driffill, J., and Martin Sola, 1999, "Policy Changes and Target Zones for Exchange Rates: the DM/FF rate 1989-1992," unpublished paper.

[7] Flood, Robert P., and Peter M. Garber, 1991, "The Linkage between Speculative Attack and Target Zone Models of the Exchange Rate," Quarterly Journal of Economics, 106, 1367-1372

[8] Flood, R.P., Rose, A.K., Mathieson, D.J., 1991. An empirical exploration of exchange-rate target-zones. Carnegie-Rochester Conference Series on Public Policy $35,7-66$.

[9] Froot, K., and M. Obstfeld, 1991, "Exchange Rate Dynamics under Stochastic Regime Shifts: A Unified Approach", Journal of International Economics, 203229 . 
[10] Garratt, A., Z. Psaradakis, and M. Sola, 2001, "Reassessing Target-Zone Nonlinearities," Journal of International Money and Finance, 533-548.

[11] Garber.P.M. and L.E.O. Svensson, 1995, Handbook of International Economics, Vol III, North-Holland.

[12] Hamilton, James. D., 1994, Time Series Analysis, Princeton University Press: Princeton NJ.

[13] Miller, M and A Sutherland, 1991 "Britain's return to Gold and entry into the EMS: joining conditions and credibility" in P. Krugman and M.Miller eds., Exchange Rate Targets and Currency Bands (C.U.P) 82-106.

[14] Obstfeld., M, 1986, "Rational and Self-fulfilling Balance of Payments Crises", American Economic Review, 76, 72-81.

[15] Obstfeld, M, 1996, "Models of Currency Crises with Self-fulfilling Features," European Economic Review, 40, 1037-48.

[16] Rose, A.K., Svensson, L.E.O., 1995. Expected and predicted realignments: the FF/DM exchange rate during the EMS, 1979-93. Scandinavian Journal of Economics $97,173-200$.

[17] Sola, Martin, and J. Driffill, 1994, "Testing the Term Structure of Interest Rates using a Stationary Auto-Regression with Regime-Switching," Journal of Economic Dynamics and Control, 18, 601-628.

[18] Svensson, Lars E. O., 1992, "An interpretation of recent research on exchange rate target zones," Journal of Economic Perspectives 6(4), Fall, 119-144.

[19] Tristani, Oreste, 1994, "Variable Probability of Realignment in a Target Zone", Scandinavian Journal of Economics, 96, 1-14.

[20] Willman, A., 1987, "Speculative Attacks on the Currency with Uncertain Policy Reactions," Economics Letters, 25, 75-78.

\section{Appendix: Solution of the Nested Configura- tion.}

The exchange rate in each state is described as follows:

$$
\begin{gathered}
\widetilde{x}_{0}(h)=\left\{\begin{array}{l}
D^{L}(h), \underline{h}_{0} \leq h<\underline{h}_{1} \\
F(h), \underline{h}_{1} \leq h \leq \bar{h}_{1} \\
D^{U}(h), \bar{h}_{1}<h \leq \bar{h}_{0}
\end{array}\right\} \\
\widetilde{x}_{1}(h)=G(h), \underline{h}_{1} \leq h \leq \bar{h}_{1}
\end{gathered}
$$


Over the range $\left[\underline{h}_{1}, \bar{h}_{1}\right]$, the functions $F($.$) and G($.$) are obtained by solving simulta-$ neously equations (10) and (11). We reproduce (and relabel) below these equations for expositional purposes.

$$
\begin{aligned}
& \widetilde{x}_{0}(h)=h+\alpha\left(\mu_{h 0} \widetilde{x}_{0 h}+\frac{\sigma_{h 0}^{2}}{2} \widetilde{x}_{0 h h}+\theta\left(\widetilde{x}_{1}(h)-\widetilde{x}_{0}(h)\right)\right) \\
& \widetilde{x}_{1}(h)=h+\alpha\left(\mu_{h 1} \widetilde{x}_{1 h}+\frac{\sigma_{h 1}^{2}}{2} \widetilde{x}_{1 h h}+\phi\left(\widetilde{x}_{0}(h)-\widetilde{x}_{1}(h)\right)\right)
\end{aligned}
$$

Equations, (A1) and (A2), are non-homogeneous and therefore their solution consists of the sum of two parts: the general solution of the homogeneous part of the equations, and the particular integrals. The homogeneous part of these two equations may be written as an equation in the vector valued function $\widetilde{x}(h) \equiv\left[\begin{array}{l}\widetilde{x}_{0}(h) \\ \widetilde{x}_{1}(h)\end{array}\right]$, viz:

$$
\left[\begin{array}{ll}
1+\alpha \theta & -\alpha \theta \\
-\alpha \phi & 1+\alpha \phi
\end{array}\right] \widetilde{x}(h)-\left[\begin{array}{ll}
\alpha \mu_{h 0} & 0 \\
0 & \alpha \mu_{h 1}
\end{array}\right] \widetilde{x}^{\prime}(h)-\left[\begin{array}{ll}
\alpha \sigma_{h 0}^{2} / 2 & 0 \\
0 & \alpha \sigma_{h 1}^{2} / 2
\end{array}\right] \widetilde{x}^{\prime \prime}(h)=0
$$

The trial solutions for the homogeneous part take the form

$$
\widetilde{x}_{0}(h)=A e^{\lambda h} \text { and } \widetilde{x}_{1}(h)=B e^{\lambda h}
$$

Substituting these solutions in equation (A3) we obtain the following relationships:

$$
\left[(1+\alpha \theta)-\alpha \mu_{h 0} \lambda-\alpha \frac{\sigma_{h 0}^{2}}{2} \lambda^{2}\right] A=\alpha \theta B
$$

and

$$
\left[(1+\alpha \phi)-\alpha \mu_{h 1} \lambda-\alpha \frac{\sigma_{h 1}^{2}}{2} \lambda^{2}\right] B=\alpha \phi A,
$$

which gives four solutions for $\lambda$, where each $\lambda_{i}$ is determined as a function of the ratio $A_{i} / B_{i}$. The $\lambda^{\prime} s$ satisfy ${ }^{20}$

$$
\left[(1+\alpha \theta)-\alpha \mu_{h 0} \lambda-\alpha \frac{\sigma_{h 0}^{2}}{2} \lambda^{2}\right]\left[(1+\alpha \phi)-\alpha \mu_{h 1} \lambda-\alpha \frac{\sigma_{h 1}^{2}}{2} \lambda^{2}\right]=\alpha^{2} \theta \phi
$$

There are two positive and two negative solutions to the polynomial. The homogeneous solutions for $x_{0}$ and $x_{1}$ are

$$
\widetilde{x}_{0}(h)=\Sigma_{i=1}^{4} A_{i} e^{\lambda_{i} h} \quad \text { and } \quad \widetilde{x}_{1}(h)=\Sigma_{i=1}^{4} B_{i} e^{\lambda_{i} h} .
$$

\footnotetext{
${ }^{20}$ The $\lambda^{\prime} s$ that satisfy this will be quite close to the ones that would satisfy [ $(1+\alpha \theta)-\alpha \mu_{h 0} \lambda-$ $\left.\alpha \frac{\sigma_{h 0}^{2}}{2} \lambda^{2}\right]=0$ and $\left[(1+\alpha \phi)-\alpha \mu_{h 1} \lambda-\alpha \frac{\sigma_{h 1}^{2}}{2} \lambda^{2}\right]=0$ for small values of $\alpha^{2} \phi \theta$.
} 
The particular solutions to (A1) and (A2) are $e_{0}=c_{0} h+a_{0}$ and $e_{1}=c_{1} h+a_{1}$. The values for the unknowns are:

$$
c_{0}=1 ; \quad c_{1}=1 ; \quad a_{0}=\frac{\alpha\left[\mu_{h 0}(1+\alpha \phi)+\mu_{h 1} \alpha \theta\right]}{1+\alpha \phi+\alpha \theta} ; \quad a_{1}=\frac{\alpha\left[\mu_{h 1}(1+\alpha \theta)+\mu_{h 0} \alpha \phi\right]}{1+\alpha \phi+\alpha \theta} .
$$

Then the general solutions of the non-homogeneous equations are:

$$
\begin{aligned}
& F(h)=\widetilde{x}_{0}(h)=\Sigma_{i=1}^{4} A_{i} e^{\lambda_{i} h}+h+\frac{\alpha\left[\mu_{h 0}(1+\alpha \phi)+\mu_{h 1} \alpha \theta\right]}{1+\alpha \phi+\alpha \theta} ; \\
& G(h)=\widetilde{x}_{1}(h)=\Sigma_{i=1}^{4} B_{i} e^{\lambda_{i} h}+h+\frac{\alpha\left[\mu_{h 1}(1+\alpha \theta)+\mu_{h 0} \alpha \phi\right]}{1+\alpha \phi+\alpha \theta} .
\end{aligned}
$$

Over the range $\left[\underline{h}_{0}, \underline{h}_{1}\right)$ we obtain $D^{L}($.$) solving the equation which results of$ replacing $\widetilde{x}_{1}(h)$ by $\underline{x}$ in equation (A1) . This leads to the following non homogeneous equation:

$$
[1+\alpha \theta] \widetilde{x}_{0}(h)=h+\alpha\left(\mu_{h 0} \widetilde{x}_{0 h}+\frac{\sigma_{h 0}^{2}}{2} \widetilde{x}_{0 h h}+\theta \underline{x}\right) .
$$

The particular integral is $d_{a}^{L}+d_{b}^{L} h$ with

$$
d_{a}^{L}=\frac{\alpha \theta \underline{x}+\alpha \mu_{0 h}(1+\alpha \theta)^{-1}}{(1+\alpha \theta)} \text { and } d_{b}^{L}=\frac{1}{(1+\alpha \theta)},
$$

and the solution to the homogeneous part of the equation is $D_{1}^{L} e^{\delta_{1}^{L} h}+D_{2}^{L} e^{\delta_{2}^{L} h}$ where $\delta_{1}^{L}$ and $\delta_{2}^{L}$ are the roots $(\delta)$ of

$$
[1+\alpha \theta]=\alpha \mu_{h 0} \delta+\alpha \frac{\sigma_{h 0}^{2}}{2} \delta^{2}
$$

and therefore the general solution of the non-homogeneous equation is

$$
D^{L}(h)=d_{a}^{L}+d_{b}^{L} h+D_{1}^{L} e^{\delta_{1}^{L} h}+D_{2}^{L} e^{\delta_{2}^{L} h}
$$

with the constants of integration $D_{1}^{L}$ and $D_{2}^{L}$ chosen so as to satisfy the boundary conditions.

Over the range $\left(\bar{h}_{1}, \bar{h}_{0}\right]$, the solution for $D^{U}(h)$ is found in a similar way. We obtain that

$$
D^{U}(h)=d_{a}^{U}+d_{b}^{U} h+D_{1}^{U} e^{\delta_{1}^{U} h}+D_{2}^{U} e^{\delta_{2}^{U} h}
$$

with $d_{a}^{U}=\frac{\alpha \theta \bar{x}+\alpha \mu_{0 h}(1+\alpha \theta)^{-1}}{(1+\alpha \theta)}$ and $d_{b}^{U}=\frac{1}{(1+\alpha \theta)}$, and $\delta_{1}^{U}=\delta_{1}^{L}$, and $\delta_{2}^{U}=\delta_{2}^{L}$. 


\subsection{Terminal Conditions - Nested Bands}

In order to solve fully for the exchange rates, it is necessary to apply a number of familiar value matching and smooth pasting conditions, which apply at the points where the exchange rate touches the edges of the bands. ${ }^{21}$ The usual conditions are modified to allow for there being two states of the world. At the edges of the band, the slope of the functions $D^{L}(h)$, and $D^{U}(h)$ in state 0 and $G(h)$ in state 1, which emerge from (A4) and (A5) have to be equal to zero (smooth pasting) and the values of the functions have to equal the upper and lower points of the band (value matching). In addition, in state 0 (assumed to be the state which involves a wider bandwidth in terms of fundamentals) it is necessary that at the boundary points for state $1\left(\underline{h}_{1}, \bar{h}_{1}\right)$ the functions $D^{L}($.$) and D^{U}($.$) have the same value and slope as F($.$) .$ Therefore the conditions are:

at $\underline{h}_{0}: D^{L}\left(\underline{h}_{0}\right)=\underline{x} ; D^{L \prime}\left(\underline{h}_{0}\right)=0$,

at $\underline{h}_{1}: F\left(\underline{h}_{1}\right)=D^{L}\left(\underline{h}_{1}\right) ; F^{\prime}\left(\underline{h}_{1}\right)=D^{L \prime}\left(\underline{h}_{1}\right) ; G\left(\underline{h}_{1}\right)=\underline{x} ; G^{\prime}\left(\underline{h}_{1}\right)=0$,

at $\bar{h}_{0}: D^{U}\left(\bar{h}_{0}\right)=\bar{x} ; D^{U \prime}\left(\bar{h}_{0}\right)=0$,

at $\bar{h}_{1}: F\left(\bar{h}_{1}\right)=D^{U}\left(\bar{h}_{1}\right) ; F^{\prime}\left(\bar{h}_{1}\right)=D^{U \prime}\left(\bar{h}_{1}\right) ; G\left(\bar{h}_{1}\right)=\bar{x} ; G^{\prime}\left(\bar{h}_{1}\right)=0$.

\footnotetext{
${ }^{21}$ It is well known that it is not enough to announce intervention as a function of the exchange rate, since that leads to indeterminacy as Froot and Obstfeld (1991) showed. Rather it has to be specified in terms of the fundamentals. It is clear that it is possible to specify such intervention policies in this case. Notice also that, as soon as there has taken place an unexpected change in regime, which drives the exchange rate to the edge of the band, the government instantly intervenes discretely in order to bring the fundamental to the value that it takes at the extreme point of the band in the new regime. The discrete intervention is associated with a zero expected change in the exchange rate as in Flood and Garber (1991).
} 
Substituting the relevant expressions in the terminals conditions we obtain the following system of equations:

$$
\begin{aligned}
& d_{a}^{L}+d_{b}^{L} \underline{h}_{0}+D_{1}^{L} e^{\delta_{1}^{L} \underline{h}_{0}}+D_{2}^{L} e^{\delta_{2}^{L} \underline{h}_{0}}=\underline{x} \\
& d_{b}^{L}+\delta_{1}^{L} D_{1}^{L} e^{\delta_{1}^{L} \underline{h}_{0}}+\delta_{2}^{L} D_{2}^{L} e^{\delta_{2}^{L} \underline{h}_{0}}=0 \\
& \Sigma_{i=1}^{4} A_{i} e^{\lambda_{i} \underline{h}_{1}}+\underline{h}_{1}+\frac{\alpha\left[\mu_{h 0}(1+\alpha \phi)+\mu_{h 1} \alpha \theta\right]}{1+\alpha \phi+\alpha \theta}=d_{a}^{L}+d_{b}^{L} \underline{h}_{1}+D_{1}^{L} e^{\delta_{1}^{L} \underline{h}_{1}}+D_{2}^{L} e^{\delta_{2}^{L} \underline{h}_{1}} ; \\
& \sum_{i=1}^{4} \lambda_{i} A_{i} e^{\lambda_{i} \underline{h}_{1}}+1=d_{b}^{L}+\delta_{1}^{L} D_{1}^{L} e^{\delta_{1}^{L} \underline{h}_{1}}+\delta_{2}^{L} D_{2}^{L} e^{\delta_{2}^{L} \underline{h}_{1}} \\
& \sum_{i=1}^{4} B_{i} e^{\lambda_{i} \underline{h}_{1}}+\underline{h}_{1}+\frac{\alpha\left[\mu_{h 1}(1+\alpha \theta)+\mu_{h 0} \alpha \phi\right]}{1+\alpha \phi+\alpha \theta}=\underline{x} \text {; } \\
& \Sigma_{i=1}^{4} \lambda_{i} B_{i} e^{\lambda_{i} \underline{h}_{1}}+1=0 \text {; } \\
& d_{a}^{U}+d_{b}^{U} \bar{h}_{0}+D_{1}^{U} e^{\delta_{1}^{U} \bar{h}_{0}}+D_{2}^{U} e^{\delta_{2}^{U} \bar{h}_{0}}=\bar{x} \\
& d_{b}^{U}+\delta_{1}^{U} D_{1}^{U} e^{\delta_{1}^{U} \bar{h}_{0}}+\delta_{2}^{U} D_{2}^{U} e^{\delta_{2}^{U} \bar{h}_{0}}=0 \\
& \Sigma_{i=1}^{4} A_{i} e^{\lambda_{i} \bar{h}_{1}}+\bar{h}_{1}+\frac{\alpha\left[\mu_{h 0}(1+\alpha \phi)+\mu_{h 1} \alpha \theta\right]}{1+\alpha \phi+\alpha \theta}=d_{a}^{U}+d_{b}^{U} \bar{h}_{1}+D_{1}^{U} e^{\delta_{1}^{U} \bar{h}_{1}}+D_{2}^{U} e^{\delta_{2}^{U} \bar{h}_{1}} ; \\
& \Sigma_{i=1}^{4} \lambda_{i} A_{i} e^{\lambda_{i} \bar{h}_{1}}+1=d_{b}^{U}+\delta_{1}^{U} D_{1}^{U} e^{\delta_{1}^{U} \bar{h}_{1}}+\delta_{2}^{U} D_{2}^{U} e^{\delta_{2}^{U} \bar{h}_{1}} ; \\
& \sum_{i=1}^{4} B_{i} e^{\lambda_{i} \bar{h}_{1}}+\bar{h}_{1}+\frac{\alpha\left[\mu_{h 1}(1+\alpha \theta)+\mu_{h 0} \alpha \phi\right]}{1+\alpha \phi+\alpha \theta}=\bar{x} \text {; } \\
& \Sigma_{i=1}^{4} \lambda_{i} B_{i} e^{\lambda_{i} \bar{h}_{1}}+1=0 \text {. }
\end{aligned}
$$

There are twelve conditions to be satisfied and twelve unknowns to be solved for ( the eight constants $A_{1}, ., A_{4}, D_{1}^{L}, D_{2}^{L}, D_{1}^{U}, D_{2}^{U}$, and the four values of the ends of the bands in terms of fundamentals in each state, that is $\underline{h}_{0}, \bar{h}_{0}, \underline{h}_{1}$, and $\bar{h}_{1}$ ). The values

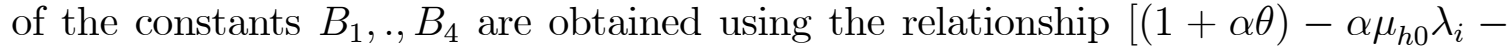
$\left.\alpha \frac{\sigma_{h 0}^{2}}{2} \lambda_{i}^{2}\right] A_{i}=\alpha \theta B_{i}$. The equations are solved numerically. 


\section{Tables and Figures}

Table 1: Derivatives of the thresholds with respect to the relevant parameters:

Nested Case

\begin{tabular}{ccccc}
\hline change in (row var.) & $\overline{h_{0}}$ & $\underline{h_{0}}$ & $\overline{h_{1}}$ & $\underline{h_{1}}$ \\
\hline change in (col. var.) & 0.0018 & -0.0018 & $9.116 e-008$ & $-8.554 e-008$ \\
$\sigma_{0}^{2}$ & $1.671 e-006$ & $-1.547 e-006$ & 0.0141 & -0.0140 \\
$\sigma_{1}^{2}$ & -0.0082 & -0.0082 & $-4.122 e-006$ & $-4.127 e-006$ \\
$\mu_{0}$ & $-4.248 e-005$ & $-4.249 e-005$ & -0.02273 & -0.0227 \\
$\mu_{1}$ & 0.0020 & -0.00208 & $2.859 e-007$ & $-3.107 e-007$ \\
$q$ & $-5.320 e-007$ & $5.983 e-007$ & -0.0044 & 0.0044 \\
$p$ & & & & \\
\hline
\end{tabular}

Table 2: Two States Estimates for Fundamentals

$$
\begin{array}{cc}
f_{t+1}=f_{t}+\alpha_{0}\left(1-s_{t+1}\right)+\alpha_{1} s_{t+1}+\left(w_{0}\left(1-s_{t+1}\right)+w_{1} s_{t+1}\right) \varepsilon_{t} \\
\hline \alpha_{0} & 0.0081 \\
& (0.0031) \\
\alpha_{1} & -0.0192 \\
& (0.0032) \\
w_{0} & 0.0146 \\
& (0.0036) \\
w_{1} & 0.0152 \\
& (0.0052) \\
p & 0.9808 \\
& (0.0232) \\
q & 0.9788 \\
& (0.0265)
\end{array}
$$

$\log L \quad 190.076$

The figures in parenthesis are standard errors.

Table 3: Derivatives of the thresholds with respect to the relevant parameters:

Overlapping Case

\begin{tabular}{ccccc}
\hline change in (row var.) & $\overline{h_{0}}$ & $\underline{h_{0}}$ & $\overline{h_{1}}$ & $\underline{h_{1}}$ \\
\hline change in (col. var.) & 0.0094 & -0.0084 & $8.914 e-007$ & $-3.729 e-007$ \\
$\sigma_{0}^{2}$ & $-1.114 e-007$ & $-1.080 e-006$ & 0.0080 & -0.01068 \\
$\sigma_{1}^{2}$ & -0.0175 & -0.0184 & $-7.187 e-006$ & $-9.997 e-006$ \\
$\mu_{0}$ & $-9.324 e-006$ & $-6.146 e-006$ & -0.0194 & -0.0172 \\
$\mu_{1}$ & -0.0044 & -0.0039 & $-1.718 e-006$ & $-2.309 e-006$ \\
$q$ & $2.300 e-006$ & $1.598 e-006$ & $0.0043-1$ & 0.0050 \\
$p$ & & & & \\
\hline
\end{tabular}




\section{Embedded Target Zone: Exchange Rate and Fundamentals}

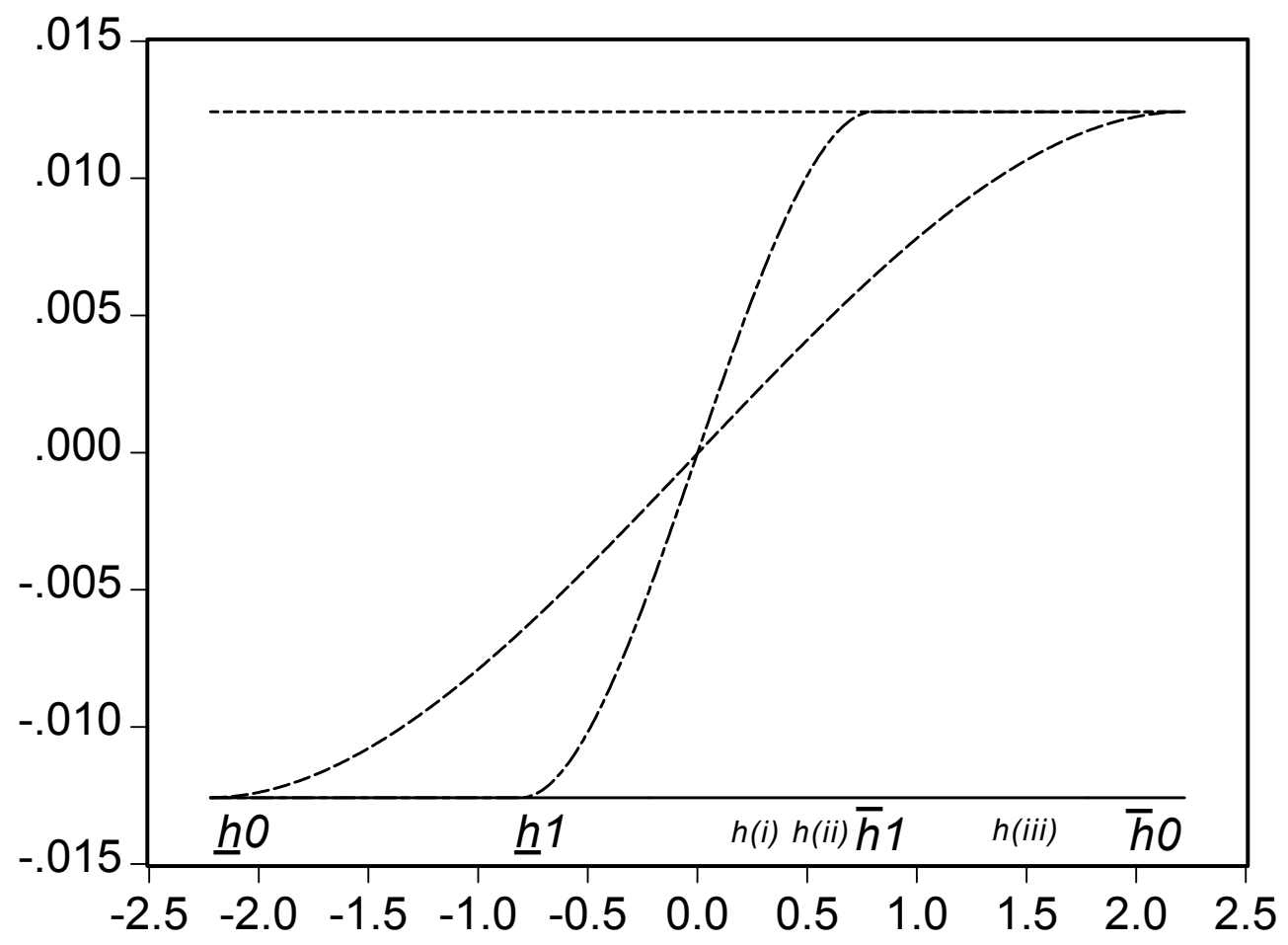

Fundamentals

Figure 1 
OverlappingTarget Zones: Exchange Rates and Fundamentals

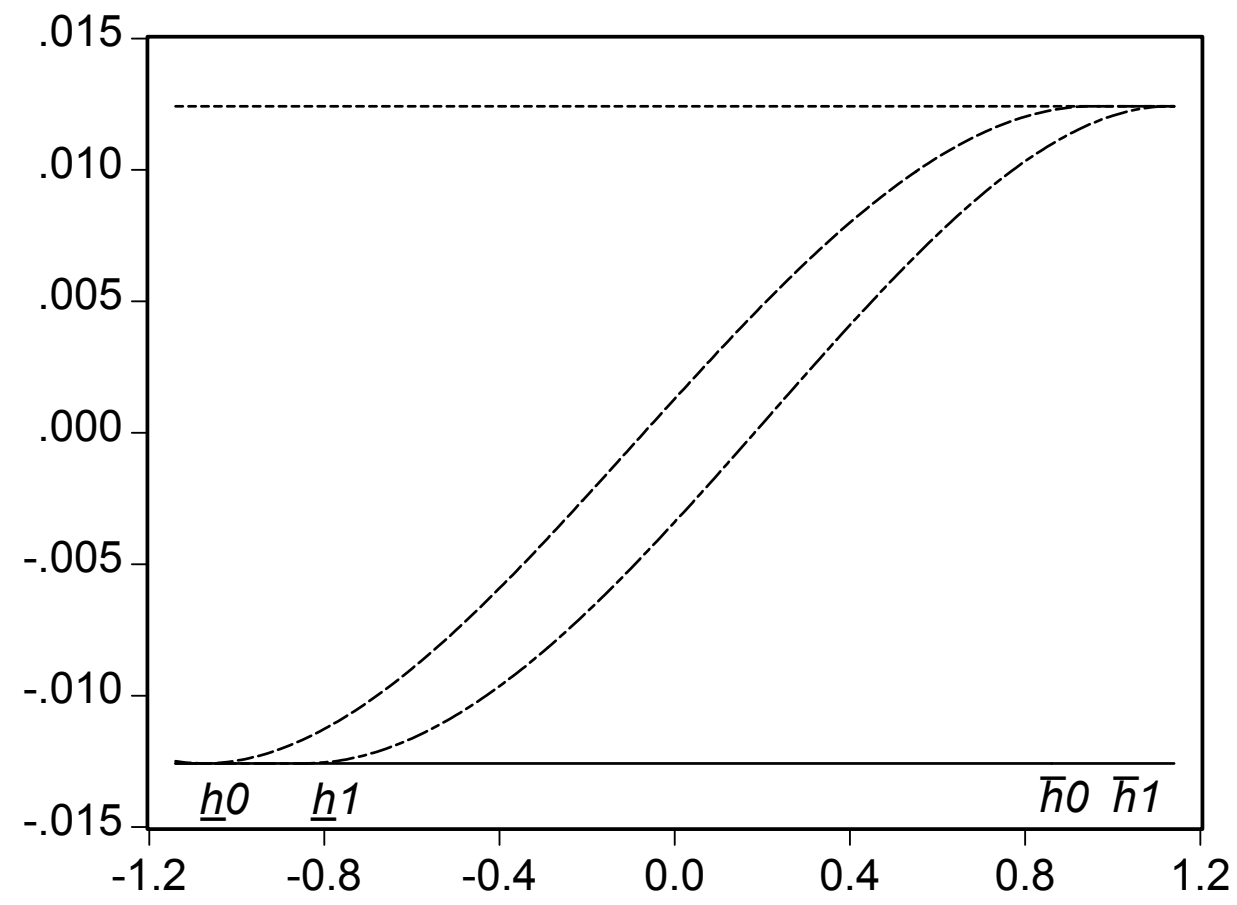

Fundamentals 
Change in Fundamentals and Probabilities of State 1

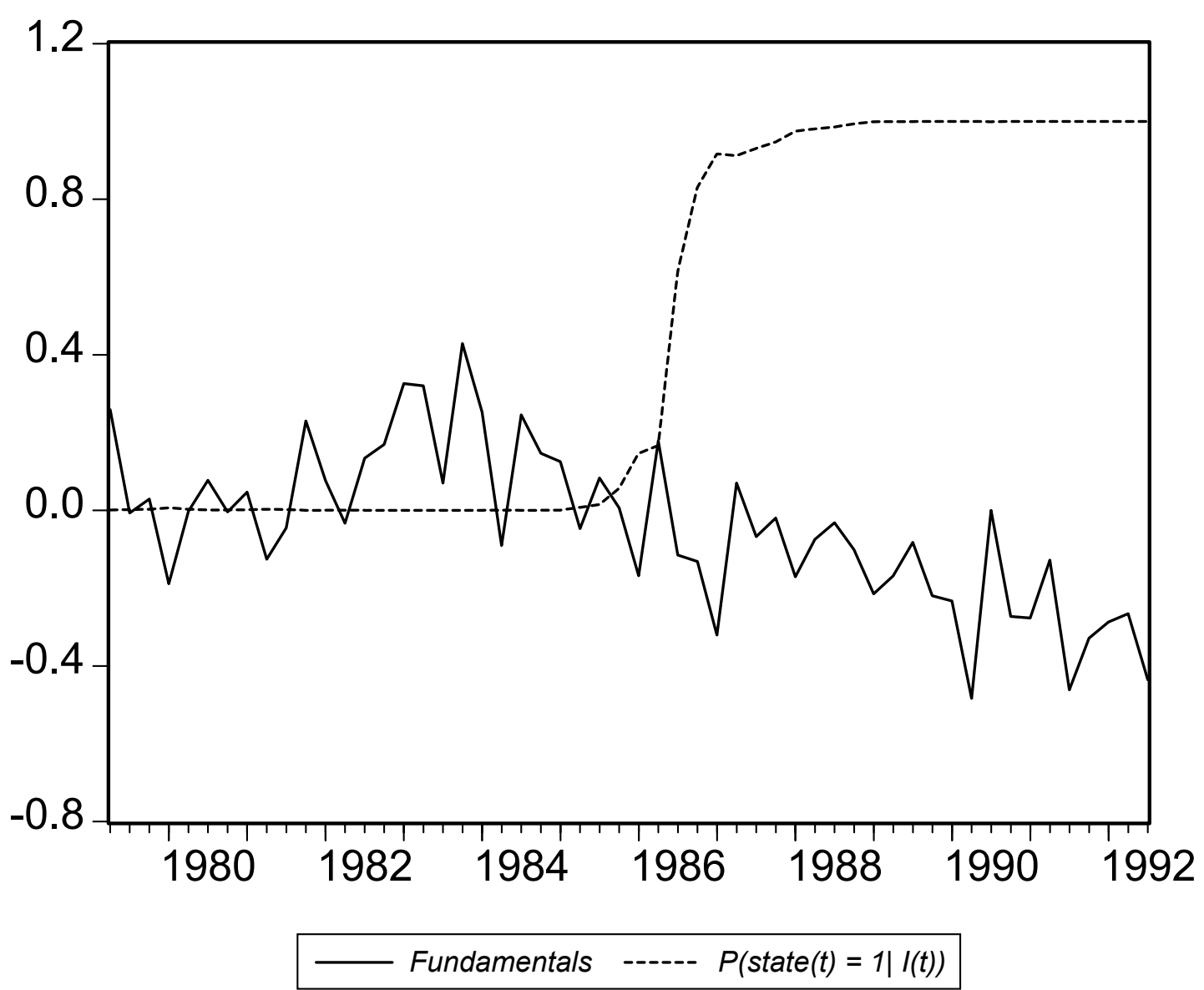

Figure 3 
Histograms of the the FR/DM Exchange Rate
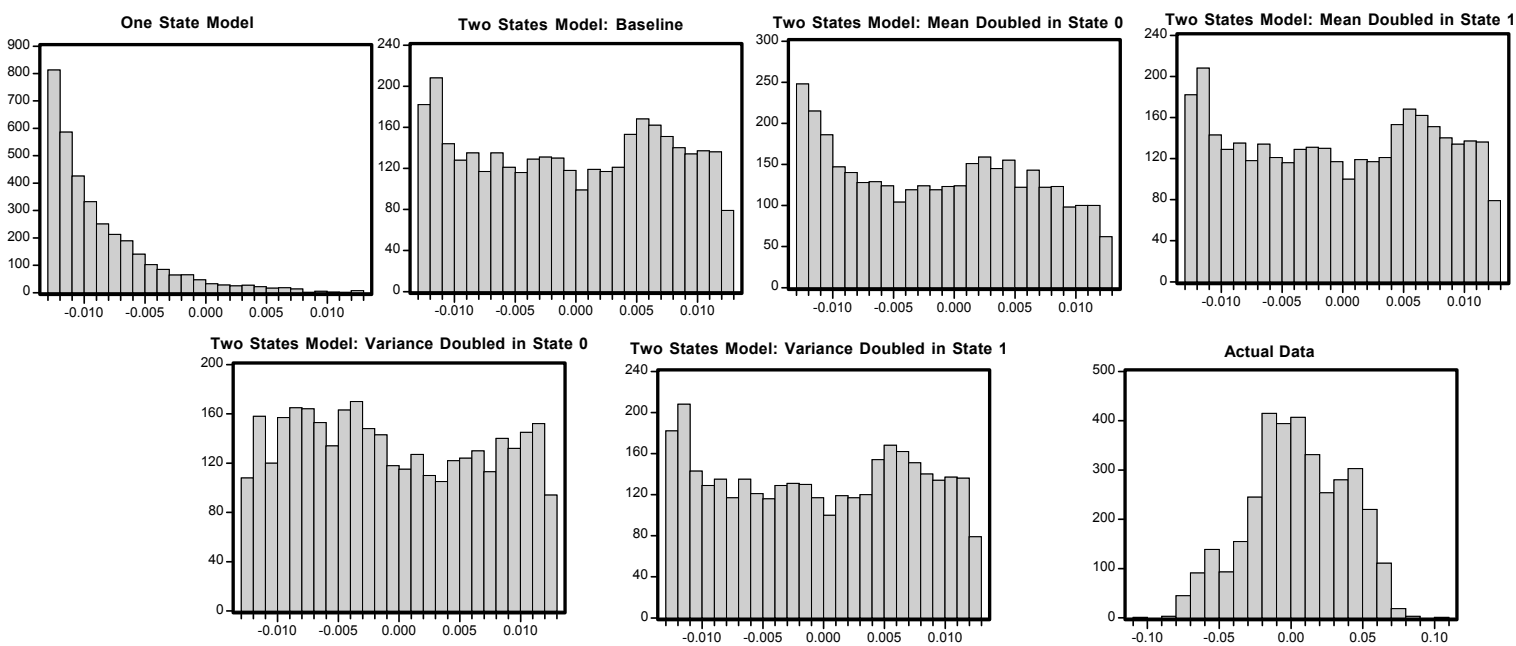

Figure 4 


\section{Interest Rate Differentials, Exchange rates and Predicted \\ Exchange Rates within the Band}
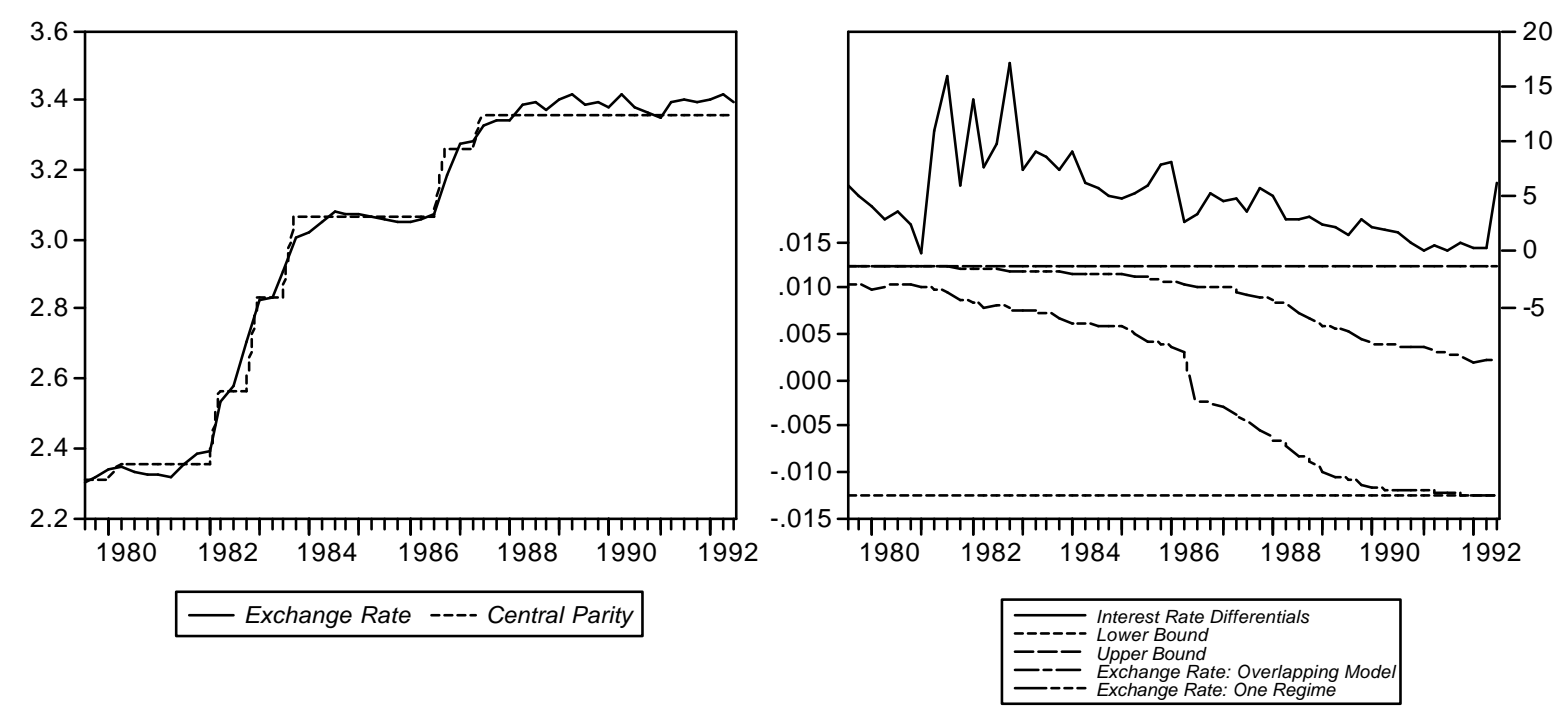

Figure 5 